\title{
STUDY OF ELECTRICAL CONDUCTIVITY OF THERMALLY REDUCED GRAPHENE OXIDE
}

\author{
Efim P. Neustroev, Mariya V. Nogovitsyna, Yuliya S. Solovyova, Grigory N. Alexandrov, \\ Evdokiya K. Burtseva
}

North-Eastern Federal University named after M.K. Ammosov, http:/ /www.s-vfu.ru

677000 Yakutsk, Russian Federation

ep.neustroev@s-vfu.ru, marianogavi88@gmail.com

\begin{abstract}
Graphene oxide under study was obtained by a modified Hummers method. Samples were subjected to thermal reduction in a temperature range from $200^{\circ}$ to $300^{\circ} \mathrm{C}$ in an atmosphere of argon and in vacuum. Results of measurements of volt-ampere characteristics of the samples in a temperature range from 80 to $300 \mathrm{~K}$ showed the presence of linear dependence of logarithm of current on a reciprocal temperature above 160-180 K. At temperatures below these values a power dependence of current on the temperature is observed. In this paper an assumption is made that both a Mott variable-range hopping (VRH) and the Efros-Shklovskii (ES-) VRH mechanism affect the electrical conductivity of graphene oxide in the range from 10 to $180 \mathrm{~K}$. A contribution of each mechanism depends on conditions of carrying out the thermal reduction.
\end{abstract}

Keywords: materials for nanoelectronics, graphene oxide, thermal reduction, current-voltage curves, temperature dependence of resistance, mechanism of electrical conductivity, thermal activation mechanism, mechanism of the variable-range hopping.

PACS: 53.039

Bibliography -17 references

Recieved 7.09.2015

RENSIT, 2015, 7(2):162-167

DOI: $10.17725 /$ rensit.2015.07.162

\section{Contens}

1. INTRODUCTION (162)

2. Materials and methods (163)

3. Results (163)

4. Discussion (164)

5. Conclusions (166)

REFERENCES (166)

\section{INTRODUCTION}

A structure of graphene oxide (GO) is represented by randomly distributed on a surface of substrate small islets of graphene with $\mathrm{sp}^{2}$ hybridized bonds surrounded by wide areas with $\mathrm{sp}^{3}$ bonds functionalized by oxygen groups [1]. In the process of reduction there are removed functional oxygen groups (epoxy, hydroxyl, carbonyl, and carboxyl) and part of crystalline structure of graphene increases [1]. One of simple and effective ways of reduction of GO is heat treatment, as a result of which one succeeds in reducing the electrical resistance of GO by several orders of magnitude [1]. The reduced graphene oxide (RGO) is inferior to graphene in its electrical and thermal properties, but in spite of that it attracts interest of many researchers. This interest is due to capabilities of use of RGO as transparent electrodes, photodetectors, elements of power sources and others [2-6].

As it is shown in papers [7-11], in the range of temperatures below $200 \mathrm{~K}$ the dependence of electrical conductivity on the temperature in the RGO is in good agreement with the hopping mechanism of conductivity with the variablerange hopping. This type of transport of charge is described by an equation $[12,13]$

$R=R_{0} \exp \left(\frac{T_{0}}{T}\right)^{m}$,

where $T$ is the temperature, $T_{0}$ is a characteristic temperature, $m$ depends on a system dimension and takes on values $1 / 3$ or $1 / 4$ - two-dimensional [9, 10] and three-dimensional [11] structures, respectively. A similar dependence with a power $m=1 / 2$ is observed for the Efros-Shklovskii mechanism [8, 11]. At higher temperatures the dependence of resistance has an activation 
character and is described by an exponential dependence [7]

$$
R=R_{0} \exp \left(\frac{E_{a}}{k_{B} T}\right),
$$

where $E_{a}$ is the activation energy and $\kappa_{B}$ is the Boltzmann constant.

When electronic devices on the basis of RGO are created, it is necessary to know influence of external factors on properties of material. One of main parameters of many devices is the electrical conductivity. The aim of this work was the study of effect of temperature on the electrical conductivity of thermally reduced GO obtained by the modified Hummers method.

\section{MATERIALS AND METHODS}

The studied graphene oxide was obtained by the modified Hummers method [14]. As a starting raw material to obtain a suspension of graphene oxide it was used graphite of company Sigma Aldrich. The suspensions of GO were subjected to ultrasonic treatment and centrifugation. As a result a homogeneous solution with average lateral dimensions of flakes of GO in a range of 0.4$0.8 \mu \mathrm{m}$ was obtained. The aqueous solution of GO was deposited on a surface of $\mathrm{SiO}_{2}$ with a thickness of $300 \mathrm{~nm}$ and was rolled by a metal rod to give uniformity in the thickness. The suspension of GO deposited in this way was evaporated at the room temperature during twenty-four hours. Silicon of $p$-type conductivity was a substrate for $\mathrm{SiO}_{2}$. Heat treatments for the reduction were performed in a temperature range from $200^{\circ}$ to $350^{\circ} \mathrm{C}$ of duration up to a few hours in an atmosphere of argon (Table 1). Measurements by methods of atomic force microscopy (AFM) and Raman spectroscopy (RS) were carried out with the help of a measuring complex "Ntegra Spectra" of company "NT-MDT".

Raman spectra of samples were measured using an excitation wavelength of $514 \mathrm{~nm}$. Radiation

Table 1

Processing conditions of sample

\begin{tabular}{|l|c|}
\hline \multicolumn{1}{|c|}{ № of sample } & Treatment temperature, ${ }^{\circ} \mathrm{C}$ \\
\hline OG-6 & 200 \\
\hline OG-1, OG-2, OG-3, OG-7 & 250 \\
\hline OG-4, OG-5 & 350 \\
\hline
\end{tabular}

power in a beam did not exceed 2-3 $\mathrm{mW}$ to prevent overheating of the sample. Also the surfaces of samples were studied with the help of scanning electron microscope of high resolution Jeol JSM 7800F. Measurements of electrical parameters were carried out on an apparatus ASEC-03 by a dual probe method in the temperature range from $80 \mathrm{~K}$ to $300 \mathrm{~K}$. The current-voltage (IV) characteristics of GO were measured in a voltage range from -10 to $+10 \mathrm{~V}$ after the reduction in the temperature range from 80 to $300 \mathrm{~K}$. Before the reduction the samples showed dielectric properties with resistances greater than $10 \mathrm{G} \Omega$. As contacts a silver paste was used.

\section{RESULTS}

Fig. $1 a$ shows a boundary of $\mathrm{GO} / \mathrm{SiO}_{2}$ measured by the method of electron microscopy for the sample OG-7. From the results of measurements
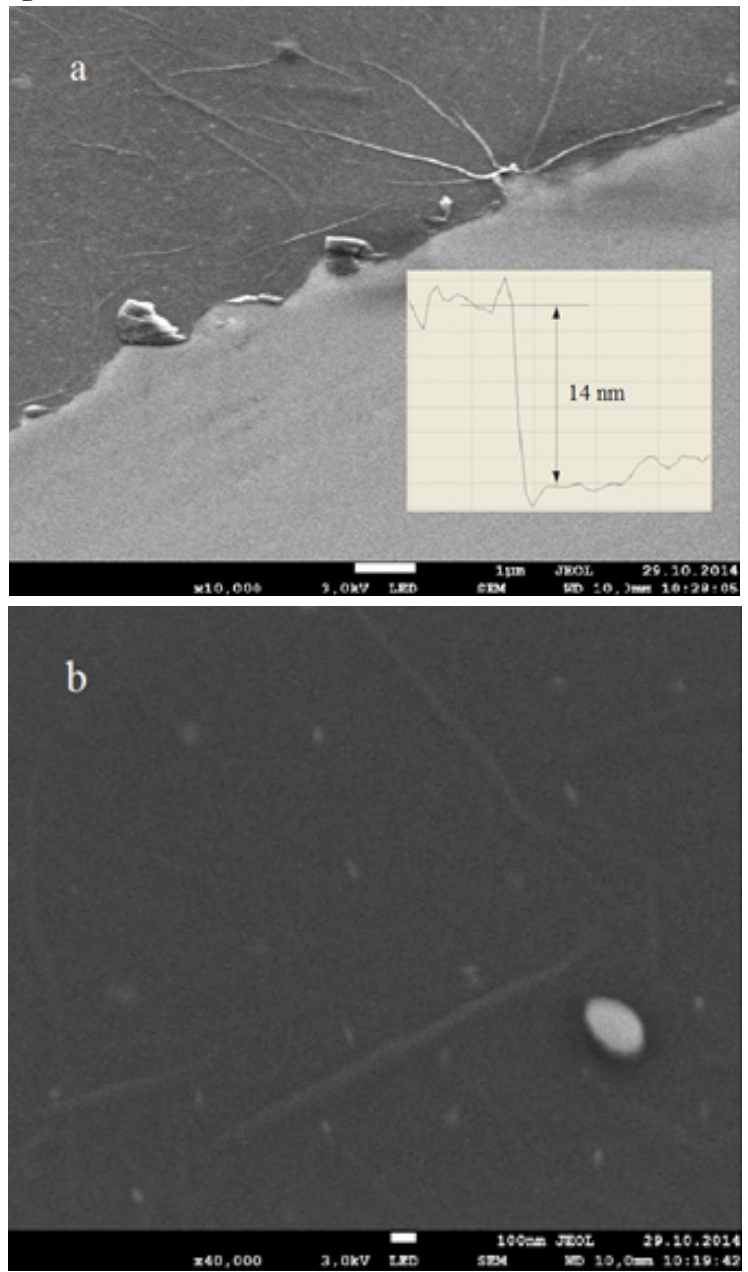

Fig. 1. Image of boundary of $\mathrm{GO} / \mathrm{SiO},(a)$ and an enlarged image of the surface (b) of sample O'G-7 after reduction obtained by scanning electron microscope. On inset of figure it is shown average value of height of step on boundary of GO/ $\mathrm{SiO}_{2}$ measured with $A F M$. 
it follows that GO is a film of relatively uniform thickness. Measurements of thickness of samples with the help of atomic force microscopy showed that the thickness of films of GO after the reduction decreases from $10-20 \mathrm{~nm}$ to $5-6 \mathrm{~nm}$. As it is seen from the Fig. $1 b$ a domain structure on the surface of GO does not become apparent. This means that sizes of graphene flakes do not exceed $10 \mathrm{~nm}$.

In the Raman spectra of all studied samples there appear typical for RGO $D$ and $G$ peaks with maxima in the neighborhood of 1350 $\mathrm{cm}^{-1}$ and $1600 \mathrm{~cm}^{-1}$, respectively. The Raman spectra of the sample OG-1 before and after the reduction are shown in Fig. 2. The peak $D$ is due to disorder of crystal lattice and formation of $s p^{3}$ bonds, the peak $G$ is due to the presence of $s p^{2}$ bonds of carbon in the lattice of graphene $[15,16]$. Relationships of intensities of peaks $I_{D} / I_{G}$ measured at different points of the sample before and after the reduction (at a temperature of $250^{\circ} \mathrm{C}$ ), leads to a slight decrease in the average value of the ratio from 0.95 to 0.90 . At the same time, a half width of peak increases by about 1.2 times.
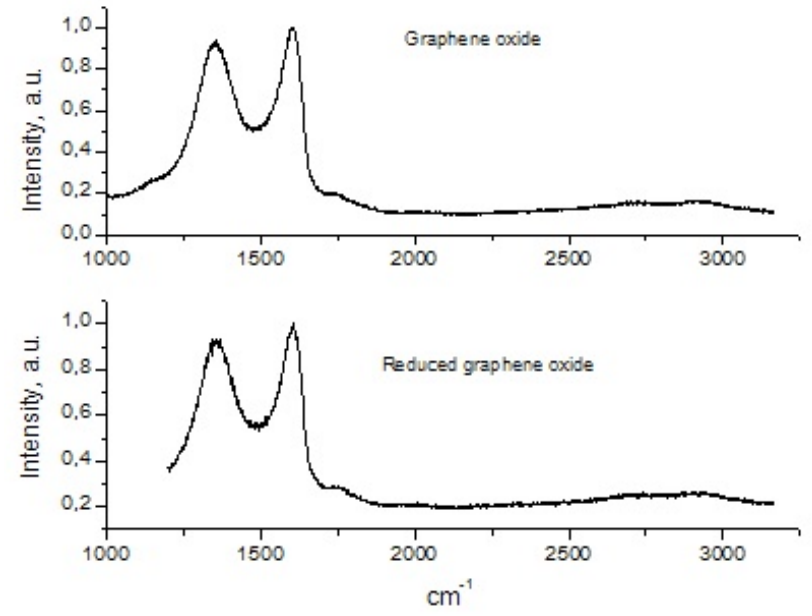

Fig. 2. Raman spectra of GO for sample OG-1 before and after reduction in atmosphere of argon at temperature of $250^{\circ} \mathrm{C}$ with duration of 30 minutes.
The IV characteristics of sample OG-1 after the reduction with duration of $1 b$ are presented in a figure (Fig. 3). The currentvoltage characteristics for all studied samples had a linear form, which indicates an ohmic nature of contacts. A difference in values of current at the temperatures of 80 and $300 \mathrm{~K}$ depends on the degree of reduction and is approximately two orders of magnitude. Sheet resistance of the samples ranged from a few to several tens of $\mathrm{k} \Omega$ per square, depending on the conditions of reduction. On an inset of the figure it is shown the dependence of logarithm of resistance on the reciprocal temperature for sample OG-1. In the figure it is possible to mark out two parts. In the high temperature part of characteristic from the room temperature to $180-220 \mathrm{~K}$ a linear dependence is observed. At lower temperatures the dependence is different from the linear one.

\section{DISCUSSION}

With the help of formula (2) the activation energies $E_{a}$ are calculated in the high temperature part of dependence of resistance on the temperature. The found values of activation energy are in a range of 0.032 to

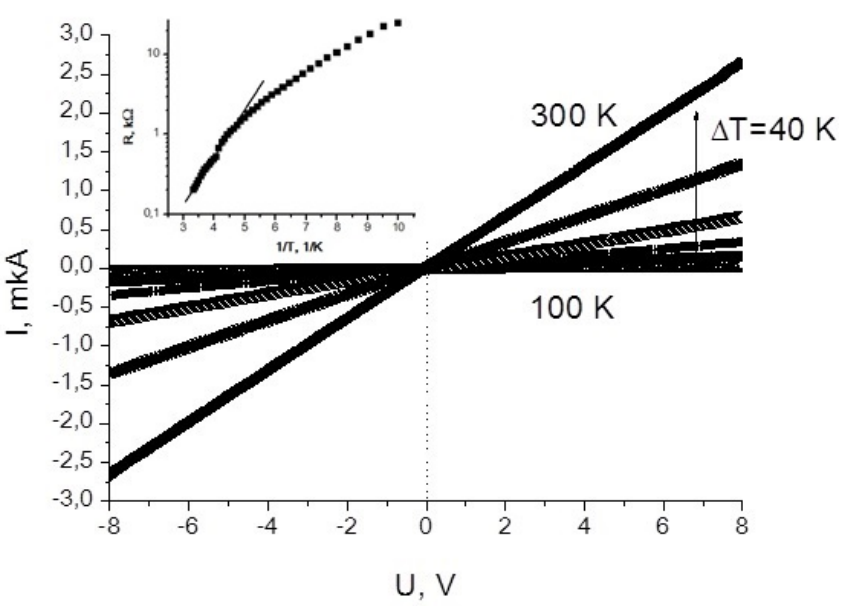

Fig. 3. Current-voltage characteristics of sample OG-1 after reduction with duration of 90 minutes. On inset of figure there is dependence of logarithm of resistance on reciprocal temperature. Line corresponds to activation mechanism of electrical conductivity at temperatures above $200^{\circ} \mathrm{C}$. 
$0.12 \mathrm{eV}$ (Table 2). As it can be seen from data of table 2, the value of energy $E_{a}$ monotonically decreases with an increase of reduction time of GO (the samples OG-2 and OG-4). Also it is found that a temperature increase of reduction up to $350^{\circ} \mathrm{C}$ leads to a more rapid decrease of the activation energy to values of $0.03-0.04 \mathrm{eV}$ (the samples OG-4, OG-5). The observed effects can be explained by transitions between states being far from the Fermi level, possibly interband transitions or states formed by functional groups of oxygen. As is known, recovery of the GO is accompanied by the departure of the various functional groups of oxygen, which can lead both to decrease the band gap [17], and the transformation of defect-impurity structure [1].

Low-temperature dependences of resistance on the temperature are different from the linear one (the inset of Fig. 3). To analyze a mechanism of conduction in this temperature range $(\Delta T)$ there are plotted the dependences of logarithm of resistance on $T^{-m}$, where $m=1 / 4,1 / 3$, and $1 / 2$, in accordance with formula (1), and there are determined coefficients of linearity $(b)$ of these dependencies. The obtained results are shown in Table 2. From an analysis of these data it follows that at temperatures of treatment of $200^{\circ}$ and $250^{\circ} \mathrm{C}$ with an increase of time of reduction a transition happens from a dependence $R(T)$ with the degree $1 / 3$ to a dependence with the degree $1 / 2$. This transition corresponds to a change of the hopping mechanism of charge transport with a variable range hopping in the two-dimensional structures to the Efros-Shklovskii mechanism $[12,13]$. At the temperature of reduction of $350^{\circ} \mathrm{C}$ already a half-hour heat treatment leads to the dependence with the degree $1 / 2$. An estimate of characteristic temperature $T_{0}$ from formula (1) gives a value of $2300 \mathrm{~K} . \mathrm{T}_{0}$ is related with the localization length of charge states $\xi$ with the help of expression [17]:

$$
T_{0}=\frac{2,8 e^{2}}{4 \pi \varepsilon \varepsilon_{0} k_{B} \xi},
$$

where $\varepsilon_{0}$ is the electric constant and $\varepsilon$ is the permittivity of the material. For the graphene oxide $\varepsilon$ is equal to 3.5 [8]. A calculation of the localization length $\xi$ according to formula (3) gives a value from 4.3 to $6.1 \mathrm{~nm}$ for samples reduced at the temperature of $350^{\circ} \mathrm{C}$ and less than $2.5 \mathrm{~nm}$ at $T=250^{\circ} \mathrm{C}$. These data agree with the data of Raman spectrum, in which a decrease of the ratio of intensities is observed, which indicates a decrease of number of edge defects caused by an increase of sizes of domains of graphene [16]. At the same time

Table 2.

Change of activation energy at temperatures above $1800 \mathrm{~K}$, change of coefficient of linearity $\mathrm{b}$ at different powers $\mathrm{m}$ in temperature range $\Delta T$, characteristic Efros-Shklovskii temperature T0 and localization length of charge states $\xi$.

\begin{tabular}{|c|c|c|c|c|c|c|c|c|}
\hline \multirow[t]{2}{*}{ Sample } & \multirow{2}{*}{$\begin{array}{c}\text { Activation } \\
\text { energy } \\
\mathrm{E}_{\mathrm{a}}, \mathrm{eV}\end{array}$} & \multirow{2}{*}{$\begin{array}{c}\text { Time of } \\
\text { reduction, } \\
\mathrm{h}\end{array}$} & \multicolumn{3}{|c|}{ linearity coefficient, b } & \multirow[t]{2}{*}{$\Delta \mathrm{T}, \mathrm{K}$} & \multirow[t]{2}{*}{$\mathrm{T}_{0}, \mathrm{~K}$} & \multirow[t]{2}{*}{$\xi, \mathrm{nm}$} \\
\hline & & & $m=1 / 4$ & $m=1 / 3$ & $m=1 / 2$ & & & \\
\hline OG-1 & 0.12 & 1.5 & 0,99877 & 0,99893 & 0,99901 & $80-180$ & 8913 & 1.5 \\
\hline OG-2 & 0.07 & 0.5 & 0,99893 & 0,99875 & 0,99824 & $80-150$ & & \\
\hline OG-2 & 0.06 & 1 & 0,99960 & 0,99960 & 0,99938 & $80-180$ & & \\
\hline OG-2 & 0.046 & 1.5 & 0,99902 & 0,99933 & 0,99975 & $90-180$ & 5200 & 2.5 \\
\hline OG-3 & & 0.5 & 0,99883 & 0,99875 & 0,99824 & $80-150$ & & \\
\hline OG-3 & & 1.5 & 0,9996 & 0,9996 & 0,99836 & $80-180$ & & \\
\hline OG-4 & 0.04 & 0.5 & 0,99872 & 0,99899 & 0,99937 & $90-175$ & 3100 & 4.3 \\
\hline OG-4 & 0.032 & 1 & 0,99894 & 0,99918 & 0,99952 & $100-175$ & 2304 & 5.8 \\
\hline OG-5 & 0.045 & 0.5 & 0,99862 & 0,99898 & 0,99948 & $85-180$ & 2206 & 6.1 \\
\hline OG-6 & 0.044 & 1 & 0,99879 & 0,99890 & 0,99877 & $80-200$ & & \\
\hline
\end{tabular}


the width $D$ of the line increases associated with an increase of disorder of lattice at the time of heat treatment [16].

\section{CONCLUSION}

Thus, it follows from the obtained results that the mechanism of conductivity in graphene oxide obtained by the chemical method and thermally reduced in the temperature range of $200^{\circ}-350^{\circ} \mathrm{C}$ in the region of temperatures above $180 \mathrm{~K}$ is determined by the activation mechanism. And the activation energy depends on the degree of reduction, which is probably related to the decrease of the width of forbidden zone. In the temperature range of 100-180 $\mathrm{K}$ there are shown the hopping mechanism of conductivity with the variable length of hop in the two-dimensional structures and the Efros-Shklovskii mechanism in the samples with a higher degree of reduction (longer treatments and a higher temperature of reduction). For this case it was carried out an estimate of sizes of domains of graphene, which reach $6.1 \mathrm{~nm}$ during the reduction at $T=350^{\circ} \mathrm{C}$ and 2.5 $\mathrm{nm}$ at $T=250^{\circ} \mathrm{C}$. The obtained results can be used to create the transparent electrodes, the photodetectors on the basis of RGO, the power sources, operating in the conditions of low temperatures.

\section{ACNOLEGMENT}

The work was performed according to the program of state task of the Ministry of education and science of the Russian Federation for higher educational institutions and scientific organizations in the sphere of scientific activity for 2014 (ST of the MES of the RF).

\section{REFERENCES}

1. Pei S, Cheng Hu-M. The reduction of graphene oxide. Carbon, 2012, 50(9):3210-3228.

2. Park W, Hu J, Jauregui LA, Ruan X, Chen YP. Electrical and thermal conductivities of reduced graphene oxide/polystyrene composites. Applied Physics Letters, 2014, 104:113101(1-4).

3. Zhu M, Li X, Guo Y, Li X, Sun P, Zang X, Wang $\mathrm{K}$, Zhong M, Wud D, Zhu H. Vertical junction photodetectors based on reduced graphene oxide/ silicon Schottky diodes. Nanossale, 2014, 6:4909-4914.

4. Lee Yu-Ying, Tu Kun-Hua, Yu Chen-Chieh, Li Shao-Sian, Hwang Jeong-Yuan, Lin Chih-Cheng, Chen Kuei-Hsien, Chen Li-Chyong, Chen Hsuen-Li, and Chen Chun-Wei. Top Laminated Graphene Electrode in a Semitransparent Polymer Solar Cell by Simultaneous Thermal Annealing/Releasing Method. ACSNano, 2011, 5(8):6564-6570.

5. El-Kadya MF, Ihns M, Li M, Hwanga JY, Mousavi MF, Chaneya L, Lecha AT, and Kaner RB. Engineering three-dimensional hybrid supercapacitors and micro supercapacitors for high-performance integrated energy storage. PNAS, 2015, 112(14):4233-4238.

6. Obreja VVN. Supercapacitors specialities Materials review. Review on Electrochemical Storage Materials and Technology. AIP Conf. Proc., 2014, 1597:98-120.

7. Muchharla B, Narayanan TN., Balakrishnan K, Ajayan PM, Talapatra S. Temperature dependent electrical transport of disordered reduced graphene oxide. 2D Materials, 2014, 1:011008(1-5).

8. Joung D, Khondaker SI. Efros-Shklovskii variable-range hopping in reduced graphene oxide sheets of varying carbon $\mathrm{sp}^{2}$ fraction. Physical Review D, 2012, 86:235423 (1-8).

9. Eda G, Mattevi C, Yamaguchi H, Kim HK, Chhowalla M. Insulator to Semimetal Transition in Graphene Oxide. J. Phys. Chem. C, 2009, 113:15768-15771.

10. Kaiser AB, Gómez-Navarro C, Sundaram RS, Burghard M, Kern K. Electrical Conduction Mechanism in Chemically Derived Graphene Monolayers. Nano Letters, 2009, 9(5):1787-1792.

11. Venugopal G, Krishnamoorthya K, Mohanc R, Kim SJ. An investigation of the electrical transport properties of graphene-oxide thin films. Materials Chemistry and Physics, 2012, 132:29-33.

12. Shklovskii BI, Efros AL. Elektronnye svojstva legirovannyh poluprovodnikov. [Electronic 
properties of doped semiconductors]. Moscow, Nauka Publ., 1979, 416 p.

13. Mott N, Devis Je. Elektronnye processy v nekristallicheskih veshhestvah. [Electronic processes in non-crystalline materials] Moscow, Mir Publ., 1982, t.1, 368 p.

14. Alexandrov GN, Smagulova SA, Kapitonov AN, Vasil'eva FD, Kurkina II, Vinokurov PV, Timofeev VB, Antonova IV. Thin Partially Reduced Oxide-Graphene Films: Structural, Optical, and Electrical Properties. Nanotecbnologies in Russia, 2014, 9(7-8):363-368 (in Russ.).

15. Diez-Betriu X, Alvarez-Garcia S, Botas C, Alvarez P, Sanchez-Marcos J, Prieto C, Menendezb R, de Andres A. Raman spectroscopy for the study of reduction mechanisms and optimization of conductivity in graphene oxide thin films. J. Mater. Chem. C, 2013, 1:6905-6912.

16. Kudin KN, Ozbas B, Schniepp HC, Prud'homme RK, Aksay IA, Car R. Raman Spectra of Graphite Oxide and Functionalized Graphene Sheets. Nano Letters, 2008, 8(1):36-41.

17. Huang H, Li Z, She J, Wang W. Oxygen density dependent band gap of reduced graphene oxide. J. Appl. Phys., 2012, 111:054317(1-4). 